\title{
NLRC4 expression in intestinal epithelial cells mediates protection against an enteric pathogen
}

\author{
S Nordlander, J Pott and KJ Maloy
}

The inflammasomes have an important role in connecting the detection of endogenous and microbial danger signals to caspase-1 activation and induction of protective immune responses. NLRC4 is a cytosolic NOD (nucleotide binding and oligomerization domain)-like receptor (NLR) that can trigger inflammasome formation in response to bacterial flagellin, an immunodominant antigen in the intestine. To characterize the role of NLRC4 in bacterially triggered intestinal inflammation, we used the murine pathogen Citrobacter rodentium, an extracellular, attaching/effacing bacterium similar to enterohemorrhagic Escherichia coli and enteropathogenic E. coli. Following infection with C. rodentium, we found that $\mathrm{NIrC4}^{-1-}$ mice developed more severe weight loss, increased bacterial colonization levels, and exacerbated intestinal inflammation compared with wild-type counterparts. NIrc4 ${ }^{-1-}$ mice mounted robust adaptive immune responses but were unable to control early colonization by $C$. rodentium, suggesting that a defect in innate immunity was responsible. Experiments using bone marrow (BM) chimeras revealed that the protective effects of NLRC4 were dependent on its expression in non-hematopoietic cells, and quantitative PCR (Q-PCR) analyses revealed that NLRC4 was highly expressed in epithelial crypts but not in intestinal stroma. Thus, early NLRC4 sensing in intestinal epithelial cells regulates colonization by an extracellular bacterial pathogen and limits subsequent intestinal damage.

\section{INTRODUCTION}

The intestinal tract is colonized by vast numbers of bacteria and is continuously exposed to a wide array of antigens and microbial products, requiring the mucosal immune system to induce tolerance to the commensal flora while still mounting potent responses to pathogens. Pattern recognition receptors (PRRs) form an integral part of the innate immune system and contribute to the first line of defense by recognizing conserved microbial patterns. ${ }^{1}$ Nucleotide binding and oligomerization domain (NOD)-like receptors (NLRs) are a group of cytosolic PRRs characterized by their central NOD, a regulatory C-terminal leucine rich repeat domain and an N-terminal domain of variable structure, such as a caspase-activation and recruitment domain. ${ }^{2}$ In response to certain microbial factors or other inflammatory stimuli, some NLRs oligomerize and form multiprotein complexes known as inflammasomes. The inflammasome serves as a platform for the activation of caspase-1, a cysteine protease, and subsequent caspase-1mediated cleavage and maturation of the pro-inflammatory cytokines interleukin (IL)-1 $\beta$ and IL-18. ${ }^{3}$ The NOD-like receptor NLRC4 forms inflammasomes following sensing of intracellular flagellin ${ }^{4,5}$ or components of type 3 bacterial secretion systems (T3SSs). ${ }^{6}$ In vitro studies using BM-derived macrophages have revealed that the NLRC4 inflammasome mediates production of active IL- $1 \beta$ in response to Salmonella typhimurium ${ }^{7}$ and limits intracellular replication of Legionella pneumophila. ${ }^{8}$ The adaptor molecule ASC (apoptosis-associated speck-like protein containing a caspase recruitment domain) has been shown to be essential for the caspase-1dependent production of the inflammasome-derived cytokines. ${ }^{9}$ However, in addition to cytokine production, activation of the NLRC4 inflammasome can result in a type of inflammasome-induced cell death termed pyroptosis, ${ }^{10}$ which can take place independently of ASC., 90

NLRC4 has been shown to contribute to protection in murine models during bacterial infection of the airways with Klebsiella pneumoniae ${ }^{11}$ and the intracellular pathogen Burkholderia pseudomallei ${ }^{12}$ by promoting survival and limiting bacterial translocation to multiple organs. ${ }^{11,12}$ Studies with the intestinal pathogen S. typhimurium have reported either redundant ${ }^{13}$ or essential protective effects ${ }^{14}$ of NLRC4, which could reflect differences in the mode of infection. Most studies 
so far have concentrated on the function of NLRC4 in hematopoietic cells, especially macrophages, and NLRC4 expression and activation in non-hematopoietic cells, such as intestinal epithelial cells (IEC), has not been well studied. However, expression of NLRP3 in non-hematopoietic cells has been proposed to be protective against dextran sulfate sodium (DSS)-induced colitis by stimulating production of IL-18. ${ }^{15}$ Further studies of inflammasome activation in IEC are needed to elucidate the interactions between the microbiota and key barrier cells in the gut. Citrobacter rodentium is a Gramnegative bacterium similar to the human pathogens enterohemorrhagic Escherichia coli (EHEC) and enteropathogenic E. coli. C. rodentium is an extracellular, attaching/effacing pathogen that adheres to the intestinal epithelium of the large intestine of mice and causes transient colonic crypt hyperplasia, inflammation, and diarrhea. ${ }^{16}$ Although infection is normally cleared in around 3 weeks, the associated pathology reproduces several facets of that seen in patients with inflammatory bowel disease. Interestingly, patients with inflammatory bowel disease have been found to harbor increased levels of adherent/invasive E.coli strains in the terminal ileum, ${ }^{17}$ suggesting a link between epithelial infection and intestinal inflammation. This makes $C$. rodentium a relevant pathogen to study in order to characterize host-pathogen interactions of the intestinal epithelium. C.rodentium colonization and pathology is dependent on the expression of a T3SS ${ }^{18}$ and T3SS components are known to trigger NLRC4 inflammasome activation in vitro, ${ }^{6}$ suggesting that NLRC4 may be activated during C. rodentium infection. Here we describe a protective role for NLRC4 against $C$. rodentium-triggered, intestinal pathology. We further show that protection is dependent on NLRC4 expression by nonhematopoietic cells, most likely IEC, which limits early bacterial colonization and subsequent intestinal inflammation. These results suggest that NLRC4 activation in IEC is a critical component of early innate defense against intestinal bacterial pathogens.

\section{RESULTS}

NLRC4 deficiency results in increased systemic immune responses to infection with enteropathogenic $C$. rodentium Although TLR-MyD88 (Toll-like receptor-myeloid differentiation primary response gene 88 ) pathways have been shown to be required for protection against $C$. rodentium, ${ }^{19,20}$ the contribution of the NLRC4 inflammasome in immune responses to extracellular, bacterial pathogens in the intestinal mucosa is not well characterized. In order to evaluate the role of the NLRC4 inflammasome, wild-type B6 (WT) and Nlrc4 ${ }^{-/-}$ mice were infected with the enteric pathogen $C$. rodentium. Susceptibility to $C$. rodentium can be affected by the intestinal microbiota in different mouse strains, which could be corrected by microbiota transplant. ${ }^{21}$ Furthermore, mice deficient in NLRP6 were more susceptible to DSS colitis, and this susceptibility could be transferred to WT mice by co-housing to allow acquisition of microbiota components. ${ }^{22}$ Therefore, in order to circumvent this potential issue, we co-housed WT and $\mathrm{NlrC}^{-/-}$mice for several weeks before infecting with
C. rodentium. In addition, we also performed C. rodentium infections in cohorts of WT and Nlrc4 ${ }^{-1-}$ mice that were housed separately and obtained identical results to those experiments where mice had been co-housed before infection.

Following oral infection with $C$. rodentium, WT mice exhibited transient diarrhea but did not lose any weight (Figure 1a). By contrast, Nlrc4 $4^{-/-}$mice suffered significant weight loss during the infection, starting approximately 8 days post infection (Figure 1a), although they eventually recovered by around 3 weeks post infection. C. rodentium is cleared via an immunoglobulin $\mathrm{G}(\mathrm{IgG})$-mediated response. ${ }^{23}$ To assess the humoral response, sera were collected from WT and Nlrc4 ${ }^{-1-}$ animals that had been infected for 3 weeks. Although WT and $N \mathrm{NrC} 4^{-1-}$ mice mounted strong C.rodentium-specific serum IgG responses, titers were significantly elevated in $\mathrm{Nlrc}^{-1-}$ animals, suggesting increased adaptive immune responses (Figure 1b). This was not due to impaired intestinal antibody responses, as levels of intestinal IgA directed at C. rodentium were similar in WT and $\mathrm{Nlrc}^{-1-}$ animals (data not shown). Consistent with the increased serum IgG titers, $\mathrm{NlrC4}^{-1-}$ animals also developed significantly elevated systemic immune activation, as shown by marked splenomegaly (Figure 1c) and increased granulocyte accumulation in the spleen (Figure 1d). However, despite the enhanced systemic responses, we rarely detected systemic C. rodentium translocation in $\mathrm{Nlrc4}^{-/-}$ mice (Supplementary Figure S1 online).

\section{NLRC4 deficiency leads to the development of severe intestinal inflammation during infection with $C$. rodentium} Following C. rodentium infection, WT mice developed mild typhlitis and moderate colitis, which peaked around day 14 post infection (Figure 2a,b). However, C. rodentium infection elicited severe inflammation in the cecum and distal colon of Nlrc4 $4^{-1-}$ mice, which was evident as early as day 8 post infection and persisted until at least day 14 post infection (Figure 2a,b). Pathological features of intestinal inflammation, including hyperplasia, leukocyte infiltration, and edema, were markedly exacerbated in $\mathrm{NlrC}^{-/-}$animals compared with the WT animals (Figure 2c). C. rodentium is known to induce epithelial cell hyperproliferation in the colon. ${ }^{19}$ In order to assess epithelial cell turnover during infection, cecal sections were stained for the cell proliferation marker Ki67. In contrast to WT mice, in which Ki67 ${ }^{+}$IEC were restricted to the basal crypt region following C. rodentium infection, $N l r c 4^{-/-}$mice clearly had increased IEC proliferation, with the proliferative zone spanning the entire length of the crypt (Figure 2d).

Effector $\mathrm{T}$ cells and cytokines, including IL-17A and interferon (IFN) $-\gamma$, are known to be induced and to mediate protection during C. rodentium infection. ${ }^{24-26}$ To assess cytokine production in the intestine, cecal tissue explants were cultured overnight. ${ }^{27}$ Levels of IFN- $\gamma$ and IL-17A in culture supernatants of uninfected animals were equivalent in WT and $\mathrm{NlrC4}^{-/-}$animals; however, Nlrc4 ${ }^{-/-}$animals had significantly elevated levels of both IFN- $\gamma$ and IL-17A, compared with WT mice after 8 and 14 days of C. rodentium infection (Figure 2e). Evaluation of effector $\mathrm{CD} 4{ }^{+}$T-cell 

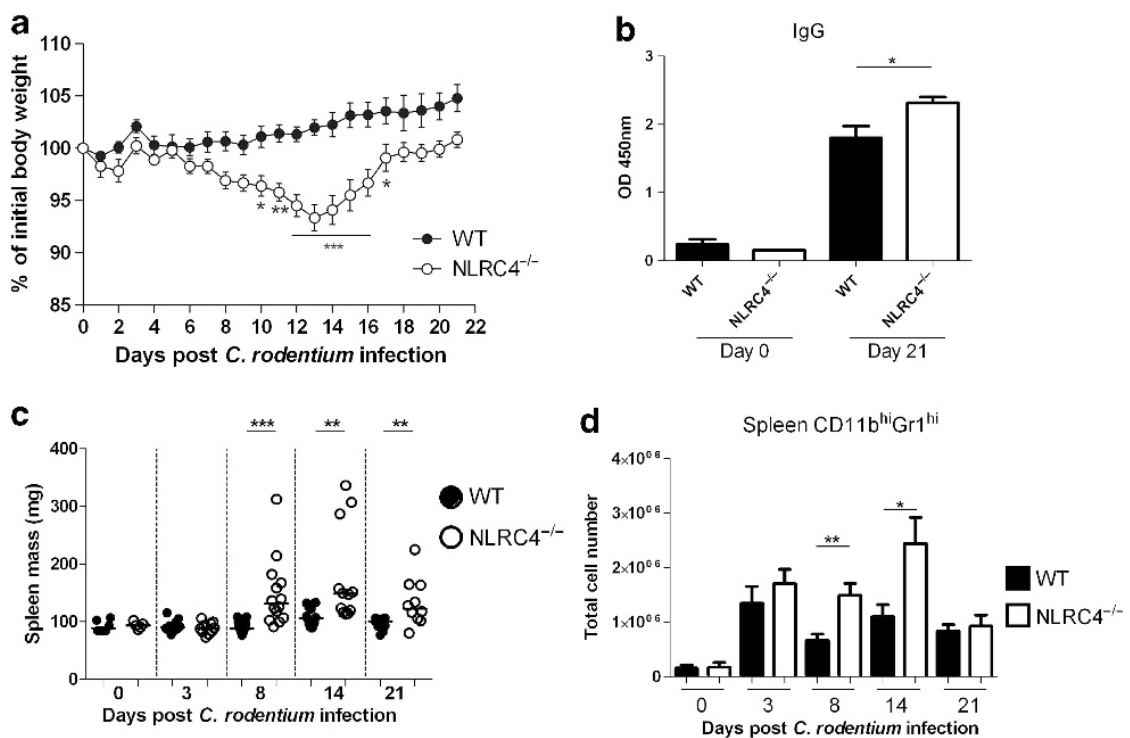

Figure 1 NLRC4 deficiency results in increased systemic immune responses during infection with enteropathogenic Citrobacter rodentium. C57BL/6 wild-type (WT) and NIrc4 ${ }^{-1-}$ mice were infected with C. rodentium. (a) Weight curve of infected C57BL/6 WT and NIrc4 ${ }^{-1-}$ mice, shown as a percentage of initial weight (data are shown as mean \pm s.e.m.; $n=10$ mice per group.) Two-way analysis of variance was performed for statistical analysis. (b) C. rodentium-specific serum IgG in infected ( $n=10$ per group) and uninfected ( $n=6$ per group) WT and NIrc4 $4^{-/-}$ mice was measured. For $\mathbf{a}$ and $\mathbf{b}$, data were pooled from two independent experiments, in which animals were infected for 21 days. (c , d) Spleen mass (c) and spleen granulocyte numbers (d) were quantified. Each symbol represents a single animal and bar graphs represent mean \pm s.e.m. Data were pooled from two independent experiments for days 3 and 21 and three independent experiments for days 8 and 14 (uninfected $n=5-6$, infected $n=10-14)$. Horizontal lines represent group medians. The non-parametric Mann-Whitney test was used for statistical analysis. ${ }^{\star} P<0.05,{ }^{\star \star} P<0.01$, ${ }^{\star \star \star} P<0.001$

accumulation in the cecal lamina propria during C.rodentium infection revealed increased T helper type 1 (Th1) and Th17 responses in $\mathrm{NlrC}^{-1-}$ mice compared with WT mice, suggesting that $\mathrm{CD}^{+}{ }^{+} \mathrm{T}$ cells were key sources of the inflammatory cytokines (Supplementary Figure S2).

We hypothesized that the excessive adaptive immune responses induced following $C$. rodentium infection of Nlrc4 ${ }^{-1-}$ mice might reflect a defect in innate immunity. To assess the role of NLRC4 in innate immunity to C. rodentium, we infected cohorts of Rag1 ${ }^{-/-}$or Nlrc4 ${ }^{-1}$ ${ }^{-} \mathrm{Rag1}^{-1-}$ animals. Compared with their NLRC4-sufficient Rag1 ${ }^{-/-}$counterparts, Nlrc4 ${ }^{-/-} \mathrm{Rag1}^{-/-}$mice developed significantly elevated intestinal inflammation and exhibited marked splenomegaly (Supplementary Figure 3A-C). Although the inflammatory response in Rag1 ${ }^{-/-}$mice was less severe than in lymphocyte-sufficient animals, the exacerbated response in $\mathrm{NlrC4}^{-/-} \mathrm{Rag1}^{-/-}$animals was preserved, suggesting that the absence of NLRC4 mainly affects the innate immune axis.

\section{NLRC4 limits bacterial colonization of the intestinal epithelium}

Increased pathology during $C$. rodentium infection has previously been linked to increased colonization levels. ${ }^{28} \mathrm{We}$ found that, relative to WT mice, Nlrc4 ${ }^{-1-}$ animals harbored significantly elevated loads of tissue-adherent $C$. rodentium in the cecum (Figure 3). Interestingly, the increased intestinal colonization in $\mathrm{NlrC}^{-/-}$mice was evident as early as day 3 and persisted until at least day 8 , but by day 14 post infection WT and Nlrc4 ${ }^{-/-}$mice had similar levels of C. rodentium (Figure 3).
These results indicate that NLRC4 limits early colonization of C. rodentium but not clearance by adaptive immunity, suggesting that it primarily participates in innate defense of the epithelium during the initial stages of infection.

\section{NLRC4 contributes to steady-state IL-18 production in the gut}

Inflammasome formation results in the cleavage and release of the leaderless pro-inflammatory cytokines IL- $1 \beta$ and IL-18. ${ }^{3}$ IL- $1 \alpha$ is not processed directly by caspase- 1 , but the release of a high-affinity species from macrophages is caspase-1 dependent and the IL-1 proteins are often co-secreted. ${ }^{29,30}$ Therefore, we assayed supernatants from organ explants of cecum and distal colon of C. rodentium-infected WT and Nlrc4 ${ }^{-1-}$ mice for IL-1 $\beta$, IL- $1 \alpha$, and IL- 18 . We found that while the IL- $1 \beta$ levels in the cecum did not fluctuate during infection (Figure 4a), there was moderate induction of IL- $1 \beta$ in the distal colon during the later stage of infection (Figure $4 \mathbf{b}$ ). However, intestinal levels of IL-1 $\beta$ were similar in WT and $N l r c 4^{-/-}$animals at all time points in both the cecum and colon (Figure $4 \mathbf{a}, \mathbf{b}$ ). We found only very low levels of IL- $1 \alpha$ in the cecum explant supernatants of $C$. rodentium-infected mice, and these were equivalent in WT and $\mathrm{Nlrc}^{-1-}$ mice during the first 8 days of infection, with $N$ lrc $4^{-/-}$mice releasing significantly more IL-1 $\alpha$ after 14 days of infection (Figure 4a). IL-1 $\beta$ is associated with granulocyte recruitment and protection from mucosal pathogens. ${ }^{11,31}$ We have previously shown that blockade of IL-1 $\beta$ reduced granulocyte recruitment to the colon during innate intestinal inflammation, ${ }^{32}$ and neutrophils are known to mediate protection during C. rodentium infection. ${ }^{33}$ However, 

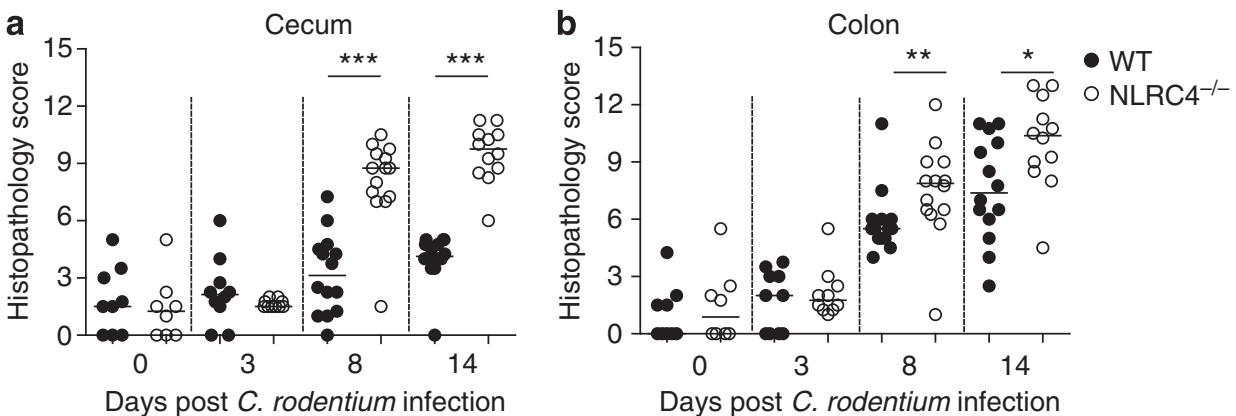

c

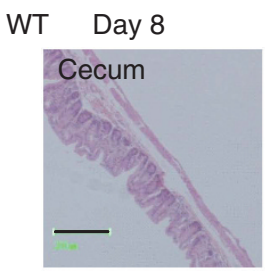

Day 14

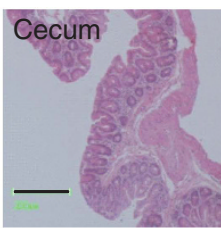

Day 14

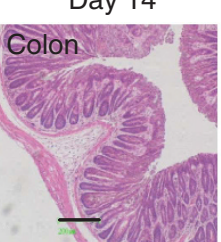

C

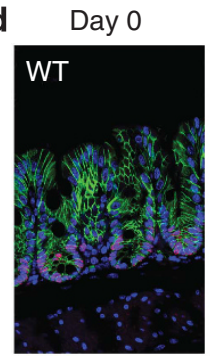

Day 8

$\mathrm{NLRC}^{-/-}$
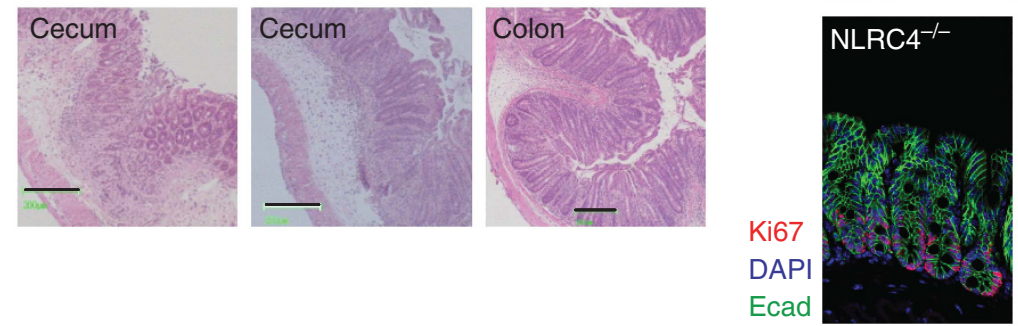

WT
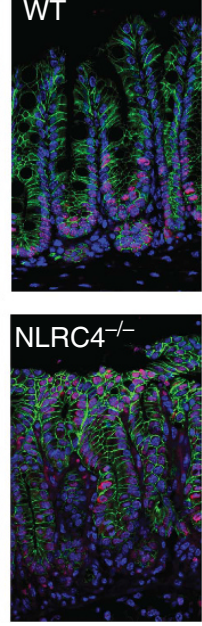

e

IFN- $\gamma$
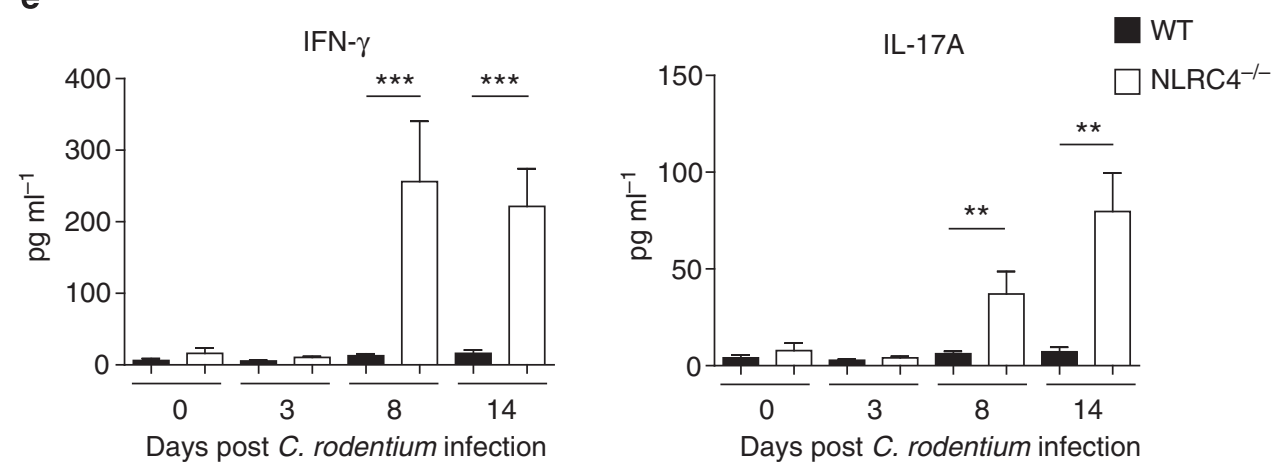

Figure 2 NLRC4 deficiency leads to the development of severe intestinal inflammation during C. rodentium infection. C57BL/6 wild-type and NIrc4 ${ }^{-1-}$ mice were infected with $C$. rodentium. Inflammation was assessed (a) in the cecum and (b) in the distal colon. Each symbol represents a single animal, and data were pooled from four independent experiments (uninfected $n=8-9$, infected $n=10-14$ ). Horizontal lines represent group medians. (c) Representative micrographs of cecum and distal colon. Black bars are equivalent to $200 \mu \mathrm{m}$. (d) Representative image of cecal tissue stained for Ki67 to assess epithelial cell turnover $\left(20 \times\right.$ objective). Sections were co-stained for E-cadherin and $4^{\prime}, 6$-diamidino-2-phenylindole (DAPI). (e) Amount of proinflammatory cytokines interferon (IFN)- $\gamma$ and interleukin (IL)-17A in cecal explant culture supernatants, normalized to tissue weight. Bar graphs represent group means \pm s.e.m. ( $n=7-8$ mice per group, pooled from two independent experiments.) The non-parametric Mann-Whitney test was used for all statistical analysis. ${ }^{\star} P<0.05,{ }^{\star *} P<0.01,{ }^{* \star} P<0.001$.

when we isolated leukocytes from cecal tissue we found that there was no impairment in neutrophil recruitment in Nlrc4 ${ }^{-1-}$ mice, as they had increased frequencies of neutrophils after infection (Supplementary Figure S4).

Analyses of IL-18 levels revealed that there was no significant increase in intestinal IL-18 in WT mice during C. rodentium infection, and IL-18 levels were comparable in WT and Nlrc $4^{-1-}$ mice throughout the infection (Figure 4c). However, we observed significantly lower IL-18 levels in cecum and colon explants from Nlrc4 ${ }^{-\prime-}$ animals compared with WT animals at steady state (Figure $4 \mathbf{c}$ ). We hypothesized that this steadystate reduction in IL-18 might contribute to the early innate immune defect in $\mathrm{NlrC}^{-1-}$ mice. In order to test this, recombinant mouse IL-18 (rIL-18) was administered daily to $N l r 4^{-1-}$ mice beginning 2 days before infection and continuing until day 6 post infection. However, we found that 


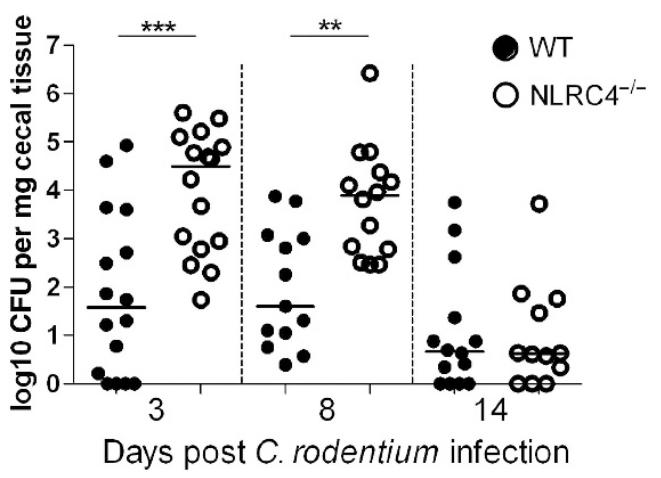

Figure 3 NLRC4 deficiency results in increased bacterial colonization. C57BL/6 wild-type and N/rc4 ${ }^{-1-}$ mice were infected with $C$. rodentium. Cecal tissue was obtained after 3,8 , and 14 days of infection, homogenized, and cultured on selective media. Colony-forming units were determined and normalized to tissue weight. Data are pooled from a minimum of three independent experiments $(n=12-16$ animals per group) per time point. Each symbol represents an individual animal, and horizontal bars represent median values. The nonparametric Mann-Whitney test was used for statistical analysis. ${ }^{\star \star} P<0.01,{ }^{\star \star \star} P<0.001$.

rIL-18-treated Nlrc4 ${ }^{-1-}$ mice harbored similar C. rodentium burdens in the cecal tissue, developed equivalent typhlitis and colitis, and displayed similar splenomegaly as control Nlrc4 ${ }^{-1-}$ mice at day 8 post infection (Supplementary Figure S5). Although these results do not definitively exclude a potential contribution of long-term NLRC4-mediated steadystate IL-18 secretion to protection from C. rodentium, they suggest that the increased susceptibility of Nlrc4 $4^{-1-}$ mice to C. rodentium infection is not simply due to a lack of IL- 1 or IL18 production. The cytokine IL- 22 has recently been shown to have a critical role in early protective responses to $C$. rodentium infection. ${ }^{34}$ This initial IL-22 is mainly produced by innate lymphoid cells $s^{35}$ and IL-22-mediated anti-bacterial responses include triggering the production of antimicrobial peptides (AMPs) Reg $3 \gamma$ and Reg3 $\beta$ and the S100 proteins S100a9 and S100a $8 .^{34,35}$ To determine whether this protective IL-22 axis was compromised in Nlrc $4^{-1-}$ mice, we assayed levels of IL-22 and AMPs in intestinal tissue. Analyses of cecal explants from WT and $\mathrm{Nlrc4}^{-/-}$mice revealed that $\mathrm{NlrC4}^{-1-}$ animals produced similar or elevated amounts of IL-22, both in steady state and throughout C. rodentium infection (Supplementary Figure S6A). Similarly, Q-PCR analyses of AMPs in cecal tissue revealed equivalent expression levels in WT and $\mathrm{Nlrc4}^{-1-}$ mice in steady state and significantly increased expression of AMPs in the intestines of $\mathrm{NlrC}^{-/-}$mice during C. rodentium infection (Supplementary Figure S6B). Together, these results indicate that Nlrc4 ${ }^{-1-}$ mice do not have any defect in IL-22 expression and mount robust AMP responses following C. rodentium infection.

\section{NLRC4 expression in non-hematopoietic cells mediates protection during C.rodentium infection}

IEC and stroma have a critical role for barrier function in the intestinal tract. IEC are known to express inflammasome components, and NLR activation has been shown to impact on host-microbiota interactions in the gut. ${ }^{22}$ In order to determine in which cell populations NLRC4 expression mediated protective effects against $C$. rodentium infection, BM chimeras were generated. Thus, cohorts of WT or Nlrc4 ${ }^{-1-}$-recipient mice were lethally irradiated and injected intravenously with $5 \times 10^{6} \mathrm{BM}$ cells from WT or Nlrc4 ${ }^{-/-}$donors. Following hematopoietic reconstitution, BM chimeras were infected with C. rodentium, and disease progression was monitored. We found that mice selectively deficient in NLRC4 expression in the non-hematopoietic cells (WT $\rightarrow \mathrm{NLRC}^{-1-}$ ) developed significant weight loss identical to that observed in complete NLRC4-deficient chimeras $\left(\mathrm{NLRC4}^{-1-} \rightarrow \mathrm{NLRC4}^{-1-}\right.$ ) (Figure 5a). Conversely, mice in which NLRC4 was selectively ablated in hematopoietic cells $\left(\mathrm{NLRC}^{-1-} \rightarrow\right.$ WT) were completely resistant to weight loss, as were control chimeras $(\mathrm{WT} \rightarrow \mathrm{WT})$ (Figure 5a). The same pattern was observed with respect to intestinal inflammation, with $\mathrm{BM}$ chimeras with a WT non-hematopoietic compartment $\left(\mathrm{WT} \rightarrow \mathrm{WT}\right.$ and $\mathrm{NLRC}^{-1-} \rightarrow \mathrm{WT}$ ) exhibiting minimal inflammation, whereas mice with NLRC4-deficient non-hematopoietic cells (NLRC4 $^{-1-} \rightarrow \mathrm{NLRC4}^{-1-}$ and $\mathrm{WT} \rightarrow \mathrm{NLRC}^{-1-}$ ) developed severe intestinal pathology (Figure 5b, c). Finally, we determined the levels of tissueadherent $C$. rodentium in the cecum and found that chimeras deficient in NLRC4 in the non-hematopoietic cells $\left(\mathrm{NLRC4}^{-1-} \rightarrow \mathrm{NLRC4}^{-1-}\right.$ and $\mathrm{WT} \rightarrow \mathrm{NLRC4}^{-1-}$ ) had significantly higher bacterial levels after 8 days of infection compared with BM chimeras harboring a WT nonhematopoietic compartment $\left(\mathrm{WT} \rightarrow \mathrm{WT}\right.$ and $\mathrm{NLRC}^{-1-} \rightarrow$ WT) (Figure 5d). The increased colonization was confirmed by immunohistochemical analyses, which revealed that the bacteria penetrated deep into the crypts in mice deficient in NLRC4 in non-hematopoietic cells (Figure 5e). In order to further define which cell types were responsible for NLRC4mediated protection, tissue fractions enriched for IECs (Crypts) and lamina propria lymphocytes (LPLs) were isolated from WT mice (Supplementary Figure S7). In addition, intestinal stromal cells (Stroma), comprising intestinal myofibroblasts were prepared. ${ }^{36}$ When assayed for NLRC4 expression, we found that IEC crypts in both the cecum and colon had the highest levels of NLRC4, significantly greater than the levels expressed by LPLs (Figure 5f). In contrast, NLRC4 could not be detected in the intestinal stroma cells (Figure 5f).

Taken together, these results clearly demonstrate that NLRC4 expression in non-hematopoietic, radioresistant cells, most likely IEC, have a critical role in protection during C. rodentium infection.

\section{DISCUSSION}

The inflammasomes have an important role in connecting endogenous and microbial danger signals to caspase- 1 activation, which in turn induces potent host immune responses that serve to restrict microbial infiltration. ${ }^{3}$ Consistent with this role in host defence, we have shown that NLRC4 is protective against infection of the intestinal tract by the non-invasive bacterial pathogen $C$. rodentium. Although a recent study reported that, compared with WT mice, Nlrc4 ${ }^{-/-}$mice had 
a

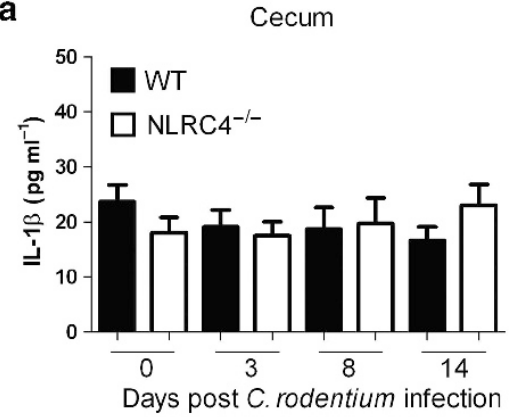

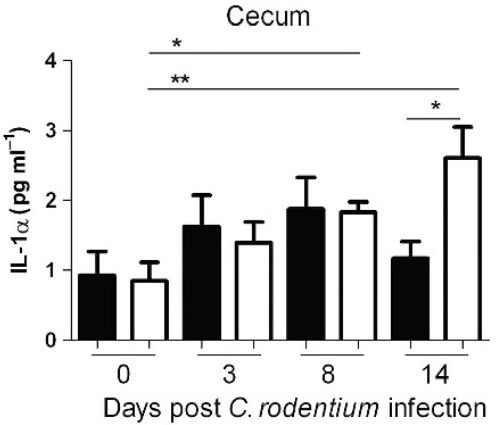

Days post C. rodentium infection

b

Colon

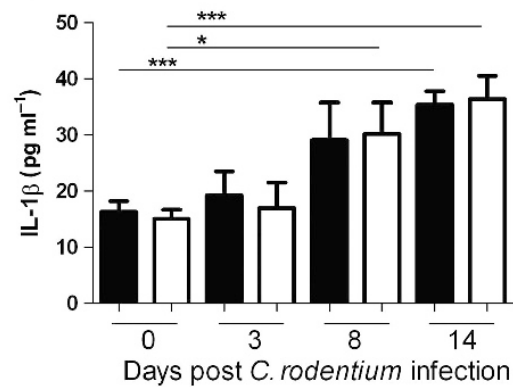

c
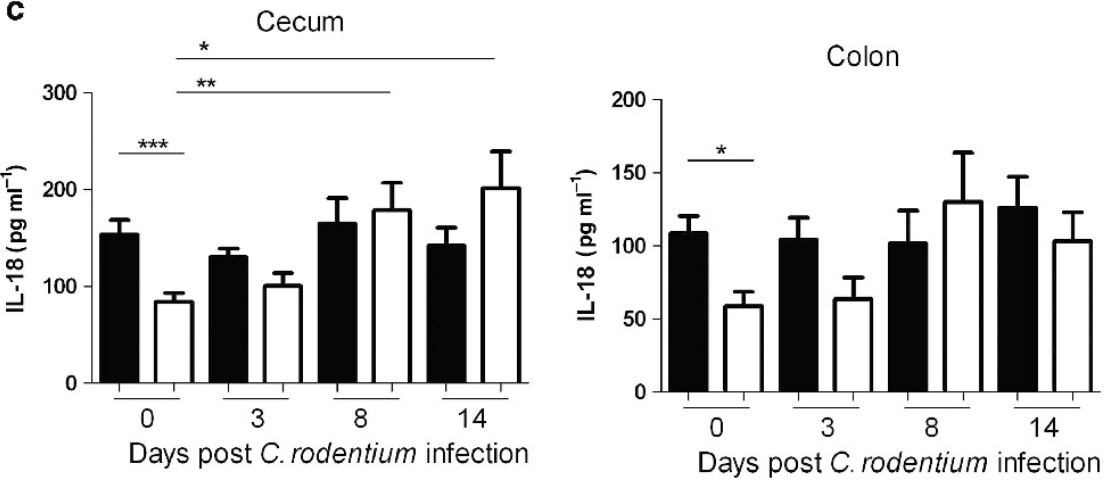

Figure 4 NLRC4 deficiency results in reduced baseline levels of intestinal interleukin (IL)-18. C57BL/6 wild-type and N/rc4 ${ }^{-/-}$mice were infected with $C$. rodentium. Tissues from cecum and distal colon were cultured overnight, and the supernatants were assayed for IL-1 and IL-18.

(a) Levels of IL-1 $\beta$ and IL-1 $\alpha$ were determined in the cecum. (b) The amount of IL-1 $\beta$ was quantified in the distal colon. (c) Amounts of IL-18 were measured in the cecum and distal colon. All values were normalized to tissue weights. For IL-1 $\beta$, bars represent pooled results from three independent experiments, $n=11-13$ animals per group mean \pm s.e.m. For IL-1 $\alpha$ and IL-18, bars represent pooled data from two independent experiments, $n=7-9$ animals per group mean \pm s.e.m. The non-parametric Mann-Whitney test was used for statistical analysis. ${ }^{\star} P<0.05,{ }^{\star \star} P<0.01$, ${ }^{* * *} P<0.001$.

significantly increased C. rodentium counts in the stool after 17 and 21 days of infection, ${ }^{37}$ our experiments highlight several novel aspects of the protection afforded by NLRC4 during C. rodentium infection. We found that NLRC4 deficiency resulted in increased bacterial adherence to the gut tissue, highly exacerbated intestinal inflammation, and elevated systemic immune activation and weight loss. Furthermore, we observed that $N l r c 4^{-1-}$ mice were rapidly colonized with $C$. rodentium during the early stage of infection, harboring approximately 1000-fold higher levels of tissue-adherent bacteria at 3 days post infection, in the absence of any apparent pathology. The increased bacterial colonization of the intestinal tissue persisted for at least 8 days, by which time the Nlrc4 ${ }^{-1-}$ mice displayed severe typhlocolitis that was associated with increased production of the inflammatory cytokines IL-17A and IFN- $\gamma$. Despite suffering from increased pathology, Nlrc4 ${ }^{-1-}$ mice mounted robust local IgA and systemic IgG responses to $C$. rodentium and eventually cleared the infection. The most straightforward interpretation of our data is that NLRC4 activation constitutes a key innate immune response that limits $C$. rodentium colonization at an early stage thus preventing subsequent immunopathology.

One critical innate immune factor that is required for protection during the early stages of $C$. rodentium infection is the cytokine IL-22. Rapid production of IL-22 by innate lymphoid cells during C. rodentium infection is essential for host survival. ${ }^{34,35}$ Colonic IECs respond to IL-22 stimulation by producing AMPs and administration of recombinant $\operatorname{Reg} 3 \gamma$, a C-type lectin that has been shown to be bactericidal in vitro, ${ }^{38}$ affords protection to IL-22-deficient animals. ${ }^{34}$ However, we 

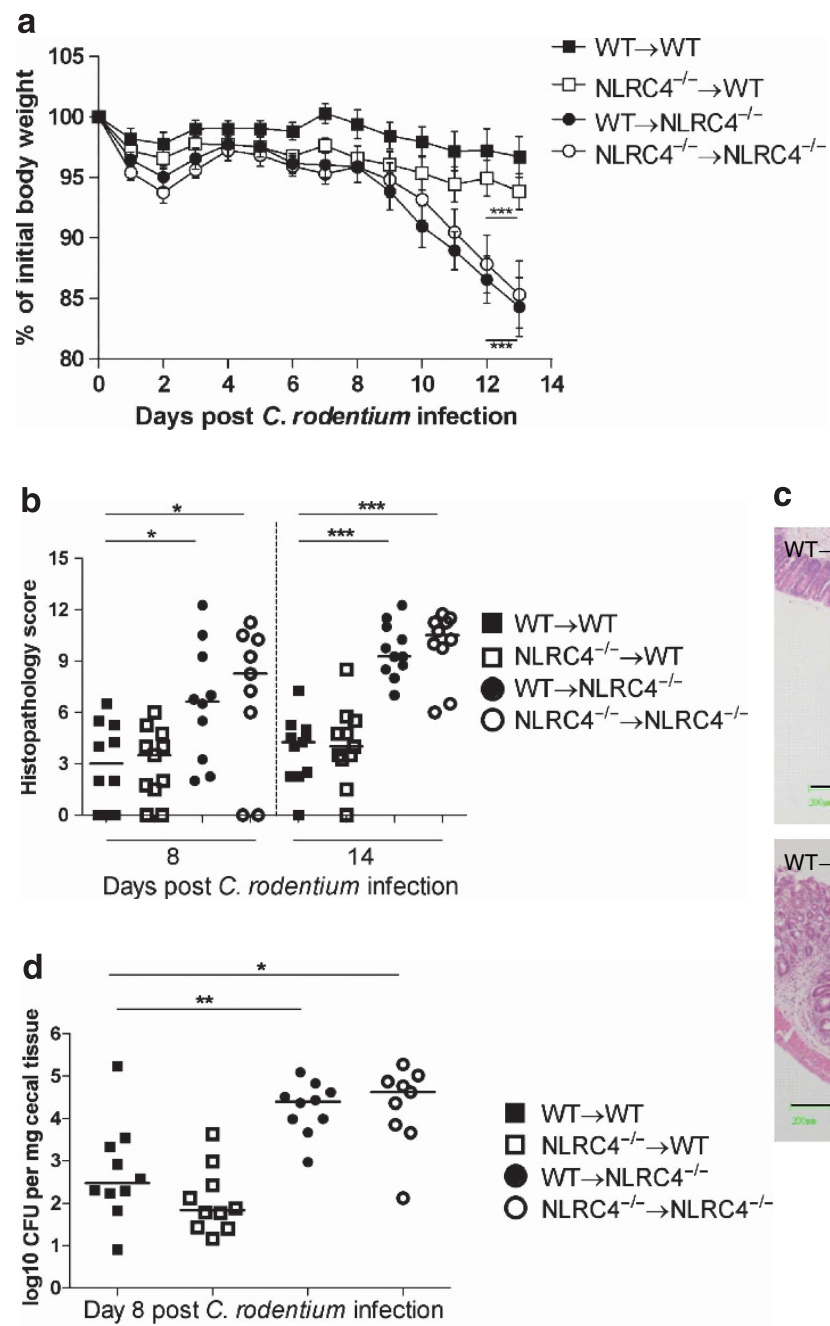

C
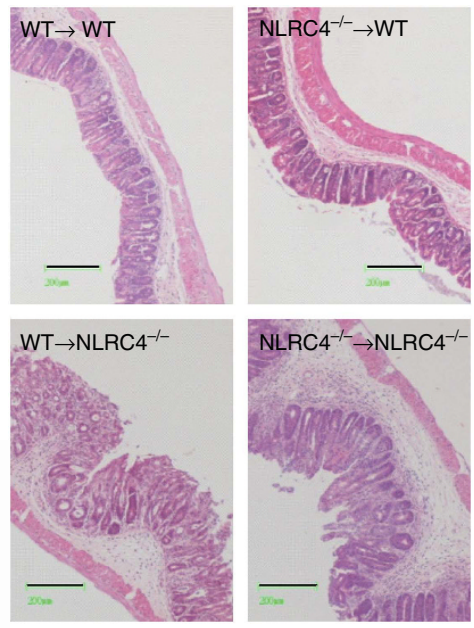

\section{ㅁ. $\mathrm{NLRC4}^{-l-} \rightarrow \mathrm{WT}$}

$\mathrm{WT} \rightarrow \mathrm{WT}$

$\mathrm{WT} \rightarrow \mathrm{NLRC} 4^{-/-}$

NLRC4 ${ }^{-/-} \rightarrow \mathrm{NLRC}^{-/-}$

Day 8 post $C$. rodentium infection

e Day 8 Cecum

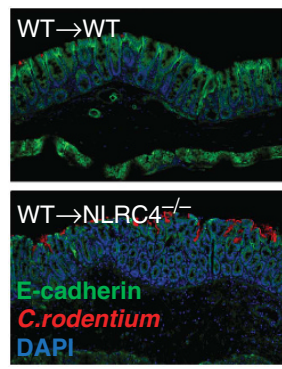

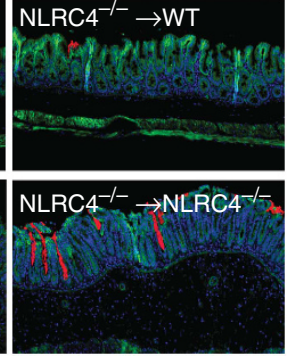

f

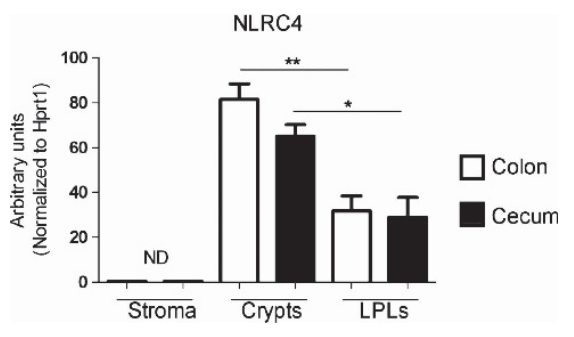

Figure 5 NLRC4 expression in non-hematopoietic cells mediates protection during $C$. rodentium infection. WT or NIrc4 ${ }^{-/-}$mice were $\gamma$-irradiated $(2 \times 5.5 \mathrm{~Gy})$ and reconstituted with $5 \times 10^{6}$ bone marrow (BM) cells isolated from either $\mathrm{NIrc}^{-/-}$or wild-type (WT) donors to generate four groups: Complete WT mice $(\mathrm{WT} \rightarrow \mathrm{WT})$; mice deficient in NLRC4 in the hematopoietic compartment (NLRC4 ${ }^{-1-} \rightarrow$ WT); mice deficient in NLRC4 in the nonhematopoietic compartment (WT $\rightarrow \mathrm{NLRC}^{-1-}$ ); and complete NLRC4-deficient animals (NLRC4 ${ }^{-1-} \rightarrow \mathrm{NLRC}^{-1-}$ ). BM chimeric mice were co-housed and left to reconstitute for 10 weeks before infection with $C$. rodentium. (a) Weight curves are shown. A number of mice (NLRC4 ${ }^{-1-} \rightarrow$ NLRC4 $^{-1-}, n=3$ and WT $\rightarrow \mathrm{NLRC}^{-1-}, n=5$ ) had to be killed earlier than 14 days post infection due to severe weight loss. Data are shown as mean \pm s.e.m.; $n=31-32$ per group at day 0 and $n=8-11$ per group at day 13 post infection. Data are pooled from two independent

experiments. Horizontal bars denote the time during which weight loss was significant. Two-way analysis of variance was used for analysis, ${ }^{* * *} P<0.001$. (b, c) Cecum pathology scores at days 8 and 14 post infection, and representative micrographs of cecum at day 14 are shown, respectively. Black bars are equivalent to $200 \mu \mathrm{m}$. (d) Cecal tissues were homogenized and plated on selective media to determine colonization levels of $C$.rodentium. Each symbol represents an individual animal, $n=9-10$ per group, pooled from two independent experiments. Horizontal lines represent group median values. (e) Representative images of $C$. rodentium-specific stain of cecal tissues at day 8 post infection $(10 \times$ objective). (f) mRNA expression levels of NLRC4 in isolated intestinal stromal cells (Stroma) and tissue fractions enriched for intestinal epithelial cells (Crypts) and lamina propria leukocytes (LPLs), respectively. Bar graphs represent means \pm s.e.m. of samples isolated from 4-6 individual mice per group. For $\mathbf{b}$, $\mathbf{d}$, and $\mathbf{f}$, the non-parametric Mann-Whitney test was used for statistical analysis. ${ }^{*} P<0.05,{ }^{* *} P<0.01,{ }^{* * *} P<0.001$. 
could determine that $\mathrm{NlrC}^{-1-}$ mice did not have any defect in IL-22 secretion either before or after infection with C. rodentium. Furthermore, Nlrc4 $4^{-1-}$ mice expressed higher levels of AMPs, including Reg3 $\gamma$, in the intestine during peak infection, confirming that they mounted strong IL-22mediated antimicrobial responses following C. rodentium infection. During C. rodentium infection, protective Th17 responses are induced, ${ }^{24,25}$ and we observed increased frequencies of Th17 cells in the intestines of infected Nlrc4 ${ }^{-1-}$ mice. Both IL-22 and IL-17A stimulation are associated with the induction of the AMPs S100A8 and S100A9. ${ }^{39}$ We found significantly increased IL-17A levels in cecal explants from infected $\mathrm{NlrC4}^{-/-}$mice, concomitant with highly elevated expression of S100a8 and S100a9, confirming that IL-17Amediated responses were not reduced, but in fact exaggerated, in susceptible animals. We hypothesize that the increased AMP responses reflect the elevated cytokine levels elicited in response to increased bacterial burdens.

NLRC4-mediated production of IL-1 $\beta$ and subsequent neutrophil recruitment has been shown to contribute to protection from $K$. pneumoniae infection of the lung, ${ }^{11}$ revealing protective effects of the NLRC4 inflammasome in a mucosal site. However, by analyzing the cellular composition of the lamina propria, we determined that $\mathrm{NlrC}^{-/-}$mice did not have delayed or reduced recruitment of neutrophils to the infected intestine. Furthermore, in the experimental $K$. pneumoniae infection noted above, protection was dependent on NLRC4 expression by hematopoietic cells. By contrast, our BM chimera experiments clearly showed that the protective effects of NLRC4 during C. rodentium infection were dependent on expression of NLRC4 in a non-hematopoietic cellular compartment. A mouse model of fungal infection of the oral cavity, using Candida albicans, revealed that NLRC4 expression in non-hematopoietic cells mediated protection by limiting colonization, systemic translocation, and pathology. ${ }^{31}$ NLRC4-deficient animals had attenuated expression of AMPs at the site of infection and reduced neutrophil recruitment to the tissue, ${ }^{31}$ which are defects in protective axes we have not observed in the $C$. rodentium model of intestinal inflammation.

We further sought to examine NLRC4 expression by fractionating the intestinal tissue. Isolated intestinal epithelial crypts showed very high expression levels of NLRC4 mRNA, while intestinal stromal cells did not express detectable NLRC4, indicating that NLRC4 expression in non-hematopoietic cells in the gut was mainly confined to IEC. Although TLR signals in IECs have been shown to contribute to maintenance of epithelial barrier function, and to protection from commensal and pathogen-induced mortality, ${ }^{40,41}$ our results indicate that intrinsic sensing of pathogenic infection by IEC may be mediated by cytosolic NLRC4. Inflammasome activation in epithelial cells is not well described, but NLRP3 expression in a non-hematopoietic compartment has been shown to be protective during DSS colitis, possibly via the production of IL-18. ${ }^{15}$ Similarly, caspase- $1^{-1-}$ mice have ablated IL-18 production and are extremely susceptible to DSS colitis but are rescued from severe pathology by the administration of recombinant IL-18. ${ }^{42}$ Previous studies have shown increased susceptibility of IL-18-deficient mice during C.rodentium infection, in that $\mathrm{IL}-18^{-1-}$ mice developed exacerbated intestinal inflammation after both 7 and 14 days of infection and had increased bacterial counts in the stool by 2 weeks. ${ }^{37,43}$ We found that although $C$. rodentium-infected WT and $N \mathrm{NrC4} 4^{-1-}$ mice expressed equivalent levels of IL-18 in the intestine, $\mathrm{NlrC4}^{-/-}$mice had lower levels of IL-18 in the tissue at steady state, suggesting that the NLRC4 inflammasome is constitutively activated in the intestine. We hypothesized that defective baseline secretion of IL-18 might explain the early innate susceptibility phenotype of the Nlrc4 ${ }^{-1-}$ mice. However, systemic administration of IL-18 did not limit colonization or ameliorate disease in $\mathrm{Nlrc} 4^{-/-}$mice. Previous studies have shown that IL-1 receptor signalling is crucial for host defence and survival during C. rodentium infection. ${ }^{43}$ However, both during steady state and following $C$. rodentium infection, we observed unaltered, low levels of intestinal IL- $1 \alpha$ and IL- $1 \beta$ secretion in the cecum of WT and Nlrc4 ${ }^{-/-}$animals. Although our results do not definitively exclude a potential contribution of inflammasome-associated cytokines to NLRC4-mediated protection against $C$. rodentium, they support the hypothesis that additional mechanisms may be triggered following activation of NLRC4 in IEC that are important for early protective immunity.

It is unclear exactly how sensing of extracellular pathogens by cytosolic PRR such as NLRC4 is accomplished. However, C. rodentium is an attaching/effacing pathogen that attaches to the intestinal epithelium through the translocated intimin receptor (Tir), which it translocates into the host cell cytosol through a T3SS. ${ }^{16}$ The NLRC4 inflammasome is formed after sensing cytosolic flagellin or components of bacterial T3SSs, and although C. rodentium is immotile, ${ }^{44}$ the T3SS could provide the agonist molecule. However, some effector proteins of the T3SS are necessary for in vivo virulence, ${ }^{18}$ making further dissection of a possible ligand challenging. Interestingly, the inflammasome-activating properties of $C$. rodentium in macrophages were reported to be independent of both a functional T3SS and NLRC4. ${ }^{37}$

A recent study reported that NLRP3 was required for protection from $C$. rodentium and this was ascribed to the secretion of IL-1 $\beta$ and IL-18 by NLRP3 inflammasomes. ${ }^{37}$ However, this study looked at the late phases of infection (from day 14 to day 21 post infection) and did not identify the cell type responsible for this protective NLRP3 response. ${ }^{37}$ Nevertheless, when taken together with our results, it can be concluded that more than one inflammasome contributes to host defense during $C$. rodentium infection and that these protective pathways are non-redundant. It is possible that distinct inflammasomes in different cell types provide protection at different phases of infection, possibly through distinct effector mechanisms. Further studies will be required to elucidate the conserved and cell-type-specific mechanisms induced upon NLR activation in IEC and intestinal leukocytes and their roles in protection and pathology in the gut. 


\section{METHODS}

Mice. C57BL/6 (B6), B6.NLRC4 ${ }^{-1-}\left(\mathrm{Nlrc4}^{-/-}\right), \mathrm{B}^{-\mathrm{RAG} 1^{-1-}}$ $\left(\right.$ Rag1 $\left.^{-1-}\right)$, and B6.NLRC4 ${ }^{-1-} \mathrm{RAG1}^{-{ }^{-}}\left(\mathrm{NlrC4}^{-/-} \mathrm{Rag1}^{-/-}\right)$ mice were bred and maintained under specific pathogen-free conditions in accredited animal facilities at the University of Oxford, Oxford, UK. Mice were at least 8 weeks of age when infected. To control for any potential micro flora differences, WT and $\mathrm{NlrC4}^{-/-}$ mice were co-housed for $\geqslant 3$ weeks before infection. ${ }^{22}$

C. rodentium infection. A nalidixic acid (NAL)-resistant strain of C. rodentium (ICC169) was grown overnight at $37^{\circ} \mathrm{C}$ in Luria broth supplemented with NAL $\left(50 \mu \mathrm{g} \mathrm{ml}^{-1}\right.$, Sigma Aldrich, Gillingham, UK). Mice were fed on average $5 \times 10^{9}$ bacteria by oral gavage. Colonization was assessed by homogenizing cecal tissue, culturing tissue homogenates on Luria broth plates supplemented with NAL, enumerating the colony-forming units recovered, and normalizing the colonization levels to tissue weight. Spleens were homogenized and cultured on selective plates. ${ }^{45}$

Evaluation of intestinal inflammation. Tissue samples of cecum and colon were fixed in buffered formalin solution (10\%) and embedded in paraffin. Five micrometer sections were then stained with hematoxylin and eosin.

C. rodentium-induced intestinal inflammation was assessed in a blinded manner using an amended version of a previously described scoring system ${ }^{32}$ in which the following five criteria were graded (from 0 to 3): degree of epithelial hyperplasia, goblet cell depletion or damage; leukocyte infiltration in the lamina propria; submucosal inflammation; area of tissue affected; and markers of severe inflammation (crypt abscesses, bleeding, necrosis). Scores were added for an overall inflammation score for each section $(0-15)$. Colon scores represent distal colon only.

Isolation and analysis of leukocyte populations. Splenocytes and leukocytes from the cecal and colonic lamina propria (LPLs) were isolated as previously described. ${ }^{36}$ Splenocytes were surface stained with e450 anti-CD45, phycoerythrin anti-CD11b, and PerCP-Cy5.5 anti-Gr1 (eBioscience, Hatfield, UK) for $15 \mathrm{~min}$ at $4{ }^{\circ} \mathrm{C}$. LPLs were isolated by incubating colonic tissue in RPMI containing fetal calf serum $(5 \%)$ and EDTA $(5 \mathrm{~mm})$ at $37^{\circ} \mathrm{C}$ in order to release IEC. The remaining tissues were digested with collagenase 8 (Sigma Aldrich) in RPMI containing fetal calf serum (5\%), DNase1 $\left(40 \mu \mathrm{g} \mathrm{ml}^{-1}\right.$, Invitrogen, Life Technologies, Paisley, UK), and HEPES (4-(2-hydroxyethyl)-1-piperazineethanesulfonic acid; $15 \mathrm{~mm}$ ) for $1 \mathrm{~h}$ at $37^{\circ} \mathrm{C}$. The LPL were then layered on a discontinuous Percoll gradient (GE Healthcare Life Sciences, Uppsala, Sweden), collected, and surface stained with fluorescein isothiocyanate anti-CD45, PerCP-Cy5.5 anti CD11b, and PeCy7 anti-Ly6G. Intracellular cytokine staining of LPL was performed as described previously. ${ }^{32}$ Briefly, LPL were restimulated for $3 \mathrm{~h}$ with $0.1 \mu \mathrm{g} \mathrm{ml}{ }^{-1}$ phorbol myristate acetate (Sigma Aldrich), $1 \mu \mathrm{g} \mathrm{ml}^{-1}$ ionomycin (Sigma Aldrich), and $10 \mu \mathrm{g} \mathrm{ml}^{-1}$ Brefeldin A (Biolegend, London, UK), then washed and stained for surface markers PeCy7 anti-CD4 and PerCP anti-TCR $\beta$. Cells were fixed overnight in Fix/Perm buffer (eBioscience) at $4{ }^{\circ} \mathrm{C}$. Cells were then washed and permeabilized in permeabilization buffer (eBioscience) with $2 \%$ rat serum for $1 \mathrm{~h}$ at $4{ }^{\circ} \mathrm{C}$. Intracellular staining was performed following permeabilization using e 450 anti-IFN- $\gamma$ and Alexa 647 anti-IL-17A or appropriate isotype controls (all from eBioscience) for $30 \mathrm{~min}$ at $4{ }^{\circ} \mathrm{C}$. Samples were analyzed on Dako Cyan (Beckman Coulter, High Wycombe, UK).

C. rodentium enzyme-linked immunosorbent assay (ELISA). Plates were coated overnight with heat-killed C. rodentium ${ }^{46}$ and blocked with bovine serum albumin (5\%). Wells were then incubated with serially diluted mouse sera, and C. rodentium reactivity was detected with biotinylated goat anti-mouse IgG and AvidinD-HRP (Vector Laboratories, Peterborough, UK). TMB substrate (eBioscience) was used, and optical density was measured at $450 \mathrm{~nm}$ using a plate reader (Molecular Devices, Wokingham, UK).

Generation of BM chimeras. BM was isolated from B6 or Nlrc4 $4^{-/-}$ mice and $5 \times 10^{6}$ cells were injected intravenously into lethally irradiated $\left(2 \times 5.5 \mathrm{~Gy}\right.$, given $4 \mathrm{~h}$ apart) B6 or Nlrc4 ${ }^{-1-}$ recipients, which were co-housed and left to reconstitute for 10 weeks. Efficient reconstitution by donor BM cells was confirmed by Q-PCR for the NLRC4 gene in splenocytes isolated from BM chimeric mice.

Isolation of intestinal crypts and stromal cells. Intestinal crypts were isolated according to a modified version of a previously published protocol. ${ }^{47}$ In brief, cecal and colonic tissues were isolated and cut into $5 \mathrm{~mm}$ pieces, which were washed with ice-cold phosphate-buffered saline multiple times and then incubated in phosphate-buffered saline with EDTA ( $25 \mathrm{~mm}$ ) with gentle agitation at $4{ }^{\circ} \mathrm{C}$ for $1 \mathrm{~h}$. Samples were washed, and supernatants enriched for intestinal crypts were collected. Isolates were examined under an inverted microscope (Leitz labovert, Leica Microsystems, Milton Keynes, UK) to confirm the presence of crypts, and purity was assessed by measuring mRNA levels of villin 1 and CD45. Intestinal stromal cells were obtained from the 30/40\% Percoll interface ${ }^{36}$ during LPL isolation and then cultured in R10 media (RPMI, 10\% fetal calf serum, HEPES, penicillin, streptomycin, and amphotericin B). After several passages, examination under an inverted microscope confirmed a homogenous cell population of fibroblast phenotype.

RNA isolation and Q-PCR. Cecal tissue, LPLs, crypts, and intestinal stromal cells were lysed in RLT buffer (Qiagen, Manchester, UK) with $\beta$-mercaptoethanol. RNA was isolated using the RNeasy kit (Qiagen), with a DNase1 digestion step. cDNA synthesis was performed using the Superscript III reverse transcription kit (Invitrogen). Real-time Q-PCR for the candidate gene was performed using SYBR green (Sensimix, Bioline Reagents, London, UK) or the TaqMan Assays system (ABI, Life Technologies, Paisley, UK). Samples were assayed in triplicate and normalized to HPRT (hypoxanthine-guanine phosphoribosyltransferase). Gene expression levels were calculated using the $\Delta$-Ct method. ${ }^{36}$ The following primer sequences were used: Nlrc4: F: $5^{\prime}$-CGGCCTGCAACCTCTTTCTT- $3^{\prime}$ and R: $5^{\prime}$-TGGGCCAAAA CATTCAGGTCT-3'. QuantiTect primer Assay was used for Hprt (Qiagen). Primer pairs and probes for Taqman: Taqman Gene Expression Assays was used for mouse Hprt, Reg3g, Reg3b, S100a8, S100a9, Ptprc, and Vil1.

Cytokine quantification. At necropsy, tissue samples from the cecum and distal colon were isolated and cultured overnight in $400 \mu \mathrm{l}$ complete RPMI 1640, and supernatants were collected for cytokine analysis. IL-1 $\beta$ was measured using sandwich ELISA with matched coating (14-7012-85 eBioscience) and detection (13-7112-85 eBioscience) antibodies. A standard curve was generated with recombinant IL-1 $\beta$ (211-11b, PeproTech, Rocky Hill, NJ) and TMB (eBioscience) was used as substrate. IL- $1 \alpha$ and IL-18 were quantified using Mouse IL- $1 \alpha$ Ready-Set-Go ELISA (eBioscience) and Mouse IL-18 Platinum ELISA (eBioscience), respectively, following the manufacturer's instructions. Other cytokines were measured with the Mouse Flowcytomix multiplex system (eBioscience), using Simplex kits for mouse IL-17A, IFN- $\gamma$, and IL-22. Samples were analyzed on a FACSCalibur (BD Biosciences, Oxford, UK).

Administration of recombinant IL-18. Mice were intraperitoneally injected with $0.5 \mu \mathrm{g}$ recombinant Mouse IL-18 (B005-5, MBL International Corporation, Woburn, MA) daily from 2 days before infection and then for 6 days following infection.

Immunohistochemistry. For immunohistochemistry, $5-\mu \mathrm{m}$ paraffin tissue sections were deparaffinized and rehydrated using xylene and ethanol. Following antigen retrieval in citrate buffer (10 mm, pH6), samples were blocked in bovine serum albumin (5\%) and normal goat serum (10\%). Sections were stained with mouse-anti-E-Cadherin (BD 
Biosciences), rabbit-anti-Ki67 (Abcam, Cambridge, UK), Alexa Fluor 488 goat anti-mouse IgG (Life Technologies), Alexa Fluor 555 goat anti-rabbit IgG (Life Technologies), rabbit anti-C. rodentium (a kind gift from Professor Gad Frankel, Imperial College, London, UK) and mounted with Vectashield mounting media with DAPI $\left(4^{\prime}, 6-\right.$ diamidino-2-phenylindole; Vector Laboratories). Images were taken using an Inverted Olympus FV1000 Confocal system (Olympus, Southend-on-Sea, UK).

Statistical analysis. Statistical analysis was performed using Prism (GraphPad Software, La Jolla, CA). The nonparametric Mann-Whitney test was used for all statistical comparisons except weight curves, which were analyzed using two-way analysis of variance. Differences were considered statistically significant at a $P$-value of $<0.05$.

SUPPLEMENTARY MATERIAL is linked to the online version of the paper at http://www.nature.com/mi

\section{ACKNOWLEDGMENTS}

We thank Dr Vishva Dixit and Genentech for providing the NIrC4 ${ }^{-1-}$ mice and $R$. Szabady for valuable advice regarding the $C$. rodentium infection protocol and for technical help. We also thank N. Srinivasan and A. Kole for technical help, B. Owens for technical advice on stromal cell isolation, G. Frankel for the C. rodentium strain, R. Stillion for histology, and the staff of University of Oxford for animal care. This work was supported by a Wellcome Trust Programme Grant (086354, K.J.M.) and an EMBO fellowship (J.P.).

\section{DISCLOSURE}

The authors declared no conflict of interest.

c 2014 Society for Mucosal Immunology

\section{REFERENCES}

1. Kumar, H., Kawai, T. \& Akira, S. Pathogen recognition by the innate immune system. In. Re.Immunol. 30, 16-34 (2011).

2. Saleh, M. The machinery of Nod-like receptors: refining the paths to immunity and cell death. Immunol. Rev. 243, 235-246 (2011).

3. Franchi, L., Munoz-Planillo, R. \& Nunez, G. Sensing and reacting to microbes through the inflammasomes. Nat. Immunol. 13, 325-332 (2012).

4. Franchi, L. et al. Cytosolic flagellin requires Ipaf for activation of caspase-1 and interleukin 1 beta in salmonella-infected macrophages. Nat. Immunol. 7, 576-582 (2006).

5. Miao, E.A. et al. Cytoplasmic flagellin activates caspase- 1 and secretion of interleukin 1 beta via Ipaf. Nat. Immunol. 7, 569-575 (2006).

6. Miao, E.A. et al. Innate immune detection of the type III secretion apparatus through the NLRC4 inflammasome. Proc. Natl. Acad. Sci. USA 107, 3076-3080 (2010).

7. Mariathasan, S. et al. Differential activation of the inflammasome by caspase-1 adaptors ASC and Ipaf. Nature 430, 213-218 (2004).

8. Amer, A. et al. Regulation of Legionella phagosome maturation and infection through flagellin and host Ipaf. J. Biol. Chem. 281, 35217-35223 (2006).

9. Broz, P., von Moltke, J., Jones, J.W., Vance, R.E. \& Monack, D.M. Differential requirement for Caspase-1 autoproteolysis in pathogeninduced cell death and cytokine processing. Cell Host. Microbe 8, 471-483 (2010).

10. Miao, E.A. et al. Caspase-1-induced pyroptosis is an innate immune effector mechanism against intracellular bacteria. Nat. Immunol. 11, 1136-1142 (2010).

11. Cai, S., Batra, S., Wakamatsu, N., Pacher, P. \& Jeyaseelan, S. NLRC4 inflammasome-mediated production of IL-1beta modulates mucosal immunity in the lung against gram-negative bacterial infection. J. Immunol. 188, 5623-5635 (2012).

12. Ceballos-Olvera, I., Sahoo, M., Miller, M.A., Del Barrio, L. \& Re, F. Inflammasome-dependent pyroptosis and IL-18 protect against Burkholderia pseudomallei lung infection while $\mathrm{IL}-1$ beta is deleterious. PLOS Pathog. 7, e1002452 (2011).

13. Broz, P., Newton, K., Lamkanfi, M., Mariathasan, S., Dixit, V.M. \& Monack, D.M. Redundant roles for inflammasome receptors NLRP3 and NLRC4 in host defense against Salmonella. J. Exp. Med. 207, 1745-1755 (2010).
14. Carvalho, F.A. et al. Cytosolic flagellin receptor NLRC4 protects mice against mucosal and systemic challenges. Mucosal Immunol. 5, 288-298 (2012).

15. Zaki, M.H., Boyd, K.L., Vogel, P., Kastan, M.B., Lamkanfi, M. \& Kanneganti, T.D. The NLRP3 inflammasome protects against loss of epithelial integrity and mortality during experimental colitis. Immunity 32, 379-391 (2010).

16. Mundy, R., MacDonald, T.T., Dougan, G., Frankel, G. \& Wiles, S. Citrobacter rodentium of mice and man. Cell. Microbiol. 7, 1697-1706 (2005).

17. Darfeuille-Michaud, A. et al. Presence of adherent Escherichia coli strains in ileal mucosa of patients with Crohn's disease. Gastroenterology 115, 1405-1413 (1998)

18. Deng, W. et al. Dissecting virulence: systematic and functional analyses of a pathogenicity island. Proc. Natl. Acad. Sci. USA 101, 3597-3602 (2004).

19. Gibson, D.L., Ma, C., Bergstrom, K.S., Huang, J.T., Man, C. \& Vallance, B.A. MyD88 signalling plays a critical role in host defence by controlling pathogen burden and promoting epithelial cell homeostasis during Citrobacter rodentium-induced colitis. Cell. Microbiol. 10, 618-631 (2008).

20. Lebeis, S.L., Bommarius, B., Parkos, C.A., Sherman, M.A. \& Kalman, D. TLR signaling mediated by MyD88 is required for a protective innate immune response by neutrophils to Citrobacter rodentium. J. Immunol. 179, 566-577 (2007).

21. Willing, B.P., Vacharaksa, A., Croxen, M., Thanachayanont, T. \& Finlay, B.B. Altering host resistance to infections through microbial transplantation. PLoS One 6, e26988 (2011).

22. Elinav, E. et al. NLRP6 inflammasome regulates colonic microbial ecology and risk for colitis. Cell 145, 745-757 (2011).

23. Maaser, C. et al. Clearance of Citrobacter rodentium requires $B$ cells but not secretory immunoglobulin A (IgA) or IgM antibodies. Infect. Immun. 72, 3315-3324 (2004).

24. Ishigame, $\mathrm{H}$. et al. Differential roles of interleukin-17A and $-17 \mathrm{~F}$ in host defense against mucoepithelial bacterial infection and allergic responses. Immunity 30, 108-119 (2009).

25. Mangan, P.R. et al. Transforming growth factor-beta induces development of the $\mathrm{T}(\mathrm{H}) 17$ lineage. Nature 441, 231-234 (2006).

26. Shiomi, H. et al. Gamma interferon produced by antigen-specific CD4 + T cells regulates the mucosal immune responses to Citrobacter rodentium infection. Infect. Immun. 78, 2653-2666 (2010).

27. Hue, S. et al. Interleukin-23 drives innate and T cell-mediated intestinal inflammation. J. Exp. Med. 203, 2473-2483 (2006).

28. Wickham, M.E. et al. Citrobacter rodentium virulence in mice associates with bacterial load and the type III effector NleE. Microbes Infect. 9, 400-407 (2007).

29. Gross, O. et al. Inflammasome activators induce interleukin-1alpha secretion via distinct pathways with differential requirement for the protease function of caspase-1. Immunity 36, 388-400 (2012).

30. Zheng, Y., Humphry, M., Maguire, J.J., Bennett, M.R. \& Clarke, M.C. Intracellular interleukin-1 receptor 2 binding prevents cleavage and activity of interleukin-1alpha, controlling necrosis-induced sterile inflammation. Immunity 38, 285-295 (2013).

31. Tomalka, J. et al. A novel role for the NLRC4 inflammasome in mucosal defenses against the fungal pathogen Candida albicans. PLoS Pathog. 7 , e1002379 (2011)

32. Coccia, M. et al. IL-1beta mediates chronic intestinal inflammation by promoting the accumulation of IL-17A secreting innate lymphoid cells and CD4(+) Th17 cells. J. Exp. Med. 209, 1595-1609 (2012).

33. Spehlmann, M.E., Dann, S.M., Hruz, P., Hanson, E., McCole, D.F. \& Eckmann, L. CXCR2-dependent mucosal neutrophil influx protects against colitis-associated diarrhea caused by an attaching/effacing lesion-forming bacterial pathogen. J. Immunol. 183, 3332-3343 (2009).

34. Zheng, $Y$. et al. Interleukin-22 mediates early host defense against attaching and effacing bacterial pathogens. Nat. Med. 14, 282-289 (2008).

35. Sonnenberg, G.F., Monticelli, L.A., Elloso, M.M., Fouser, L.A. \& Artis, D. $\mathrm{CD} 4(+)$ lymphoid tissue-inducer cells promote innate immunity in the gut. Immunity 34, 122-134 (2011).

36. Kirchberger, S. et al. Innate lymphoid cells sustain colon cancer through production of interleukin-22 in a mouse model. J. Exp. Med. 210, 917-931 (2013).

37. Liu, Z. et al. Role of inflammasomes in host defense against Citrobacter rodentium infection. J. Biol. Chem. 287, 16955-16964 (2012). 
38. Cash, H.L., Whitham, C.V., Behrendt, C.L. \& Hooper, L.V. Symbiotic bacteria direct expression of an intestinal bactericidal lectin. Science 313, 1126-1130 (2006).

39. Liang, S.C. et al. Interleukin (IL)-22 and $\mathrm{LL}-17$ are coexpressed by Th17 cells and cooperatively enhance expression of antimicrobial peptides. J. Exp. Med. 203, 2271-2279 (2006).

40. Asquith, M.J., Boulard, O., Powrie, F. \& Maloy, K.J. Pathogenic and protective roles of MyD88 in leukocytes and epithelial cells in mouse models of inflammatory bowel disease. Gastroenterology 139, 519-529. 529 e511-512 (2010).

41. Vaishnava, S., Behrendt, C.L., Ismail, A.S., Eckmann, L. \& Hooper, L.V. Paneth cells directly sense gut commensals and maintain homeostasis at the intestinal host-microbial interface. Proc. Natl. Acad. Sci. USA 105 20858-20863 (2008).

42. Dupaul-Chicoine, J. et al. Control of intestinal homeostasis, colitis, and colitis-associated colorectal cancer by the inflammatory caspases. Immunity 32, 367-378 (2010).
43. Lebeis, S.L., Powell, K.R., Merlin, D., Sherman, M.A. \& Kalman, D. Interleukin-1 receptor signaling protects mice from lethal intestinal damage caused by the attaching and effacing pathogen Citrobacter rodentium. Infect. Immun. 77, 604-614 (2009).

44. Khan, M.A. et al. Flagellin-dependent and -independent inflammatory responses following infection by enteropathogenic Escherichia coli and Citrobacter rodentium. Infect. Immun. 76, 1410-1422 (2008).

45. Ghaem-Maghami, M. et al. Intimin-specific immune responses prevent bacterial colonization by the attaching-effacing pathogen Citrobacter rodentium. Infect. Immun. 69, 5597-5605 (2001).

46. Bry, L. \& Brenner, M.B. Critical role of Tcell-dependent serum antibody, but not the gut-associated lymphoid tissue, for surviving acute mucosal infection with Citrobacter rodentium, an attaching and effacing pathogen. J. Immunol. 172, 433-441 (2004).

47. Jung, P. et al. Isolation and in vitro expansion of human colonic stem cells. Nat. Med. 17, 1225-1227 (2011). 